BIO Web of Conferences 1, 00008 (2011)

DOI: $10.1051 /$ bioconf $/ 20110100008$

(C) Owned by the authors, published by EDP Sciences, 2011

\title{
Learning new skills in Multimodal Enactive Environments
}

\author{
Benoît G. Bardy \\ Movement to Health Laboratory, EuroMov, Montpellier-1 University \\ 700 Avenue du Pic Saint Loup, 34090 Montpellier, France \\ Email:benoit.bardy@univ-montpl.fr
}

\begin{abstract}
A European consortium of researchers in movement and cognitive sciences, robotics, and interaction design developed multimodal technologies to accelerate and transfer the (re)learning of complex skills from virtual to real environments. The decomposition of skill into functional elements - the subskills - and the enactment of informational variables used as accelerators are here described. One illustration of accelerator using virtual reality in team rowing is described.
\end{abstract}

\section{Introduction}

Skill is the capacity acquired by learning to reach a specified goal in a specific task with the maximum of success and a minimum of time, energy or both. Technology such as virtual reality devices or multimodal human-machine interfaces is more and more used, obviously for financial or technological reasons, to speed up the learning of new movements or procedures. Training in technological environments becomes a new era both for trainers and trainees, allowing the elaboration of new training scenarios and training protocols. However, the positive or negative transfer effect of this technologyoriented training (i) often seems to contradict fundamental knowledge about skill learning, (ii) requires the adequate decomposition of (complex) skill into functional elements that make sense from theoretical and pragmatic point of views, and (iii) remains to be proven using careful evaluation and transfer studies. Figure 1 illustrate six technological human-machine interfaces developed within the SKILLs european project, aiming at accelerating and transferring the (re-)learning of complex coordinative skills in virtual and real environments, and for which the three above aspects were carefully addressed. Various domains of application are concerned including sport and entertainment, surgery and rehabilitation, and industrial maintenance.

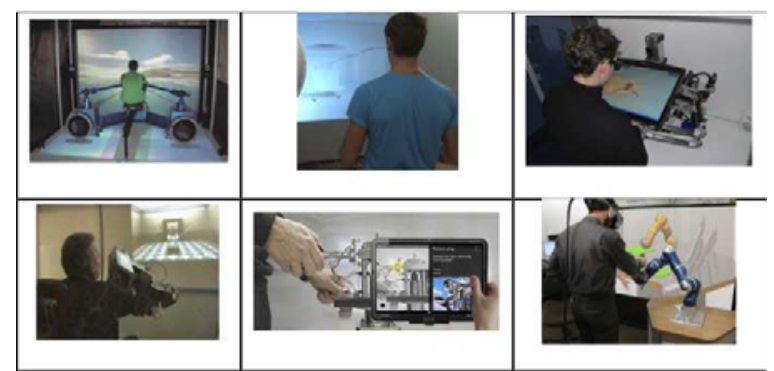

Figure 1: Six multimodal virtual reality platforms allowing the acquisition and transfer of perceptuo-motor skills in the domains of sport (rowing), entertainment (juggling), surgery (maxillofacial), rehabilitation (upperlimb), industrial maintenance (assembling/ disassembling), and programming by demonstration (prototyping). From [1]

\section{Skill decomposition}

The decomposition of complex skills in functional, (re-)trainable elements is an important step in technological training, both from theoretical and practical standpoints (see [1] for details). Elementary functional units are often described as coordinative structures [2], or motor primitives [3]. Coordinative structures are temporary (soft) assemblies of elements constrained to behave as a single functional unit, while motor primitives are force fields generated by muscle units, resulting from the stimulation of hardwire neural circuitry. Stable attractors (fixed points vs. limit cycles) are also envisaged to constitute the building blocks of (continuous vs. discrete) movements and skills [4]. Pragmatically, skill decomposition raises the question of which skill elements contributing to the global performance can be temporarily isolated from their neighbours, receive a specific training, before being incorporated again in a more general training protocol. In Table 1, the fifteen skill elements selected by the SKILLS consortium - the sub-skills - are described. They cross many of the complex skills described in Figure 1, and received a specific attention. The first nine sub-skills (upper section of Table 1) are from the sensorimotor repertoire while the last five sub-skills (lower section of Table 1) are

This is an Open Access article distributed under the terms of the Creative Commons Attribution-Noncommercial License 3.0, which permits unrestricted use, distribution, and reproduction in any noncommercial medium, provided the original work is properly cited. 
from the cognitive repertoire. In general, sensorimotor sub-skills are skills that relate to the relationship between perceptual components and motor components, and cognitive skills are related to higher-level cognitive activities that orient, formulate, monitor and regulate sensorimotor performances. The distinction is partly arbitrary as the two categories are obviously largely interdependent. It is however a convenient way to help researchers and designers to elaborate technological tools to enact and learn these skill elements.

These 15 sub-skills are general, abstract, well documented, and cross a large number of domains, from rehabilitation to surgery to industrial maintenance to sport and entertainment. For instance, bimanual coordination is a very generic (sensorimotor) sub-skill that exists both in juggling, rowing, or surgery. It includes the concatenation of limit cycle primitives spanning over several muscles and joints. Similarly, procedural sub-skills are (cognitive) components that exist both in complex surgery and industrial maintenance applications. Among a virtually infinite number of sub-skills that compose human activities, the fifteen elements described in Table 1 have been selected because of (i) their key importance for the successful achievement of skilled performance, (ii) their possible yet challenging enactment using multimodal virtual reality technology, (iii) their coverage of complementary perceptual modalities or effectors, (iv) their visible evolution over time and learning, and (v) their anchorage in a solid state-of-the-art in basic human movement and cognitive sciences. Each sub-skill is matter of theoretical and experimental research, is defined by specific variables, can be captured and rendered using specific hardware and software technologies. During the coarse of the SKILLS project, they have been investigated in several evaluation and transfer studies [5]. They have been shown to be the main components of efficient and adaptive behaviours in technology-driven learning scenarios. It is throughout the interaction of these (and other) sub-skills, under appropriate practice and training conditions, using multimodal interfaces that skilled behaviours progressively (re)emerge.
Table 1. Sensori-motor and cognitive skill elements investigated in the SKILLS european project.
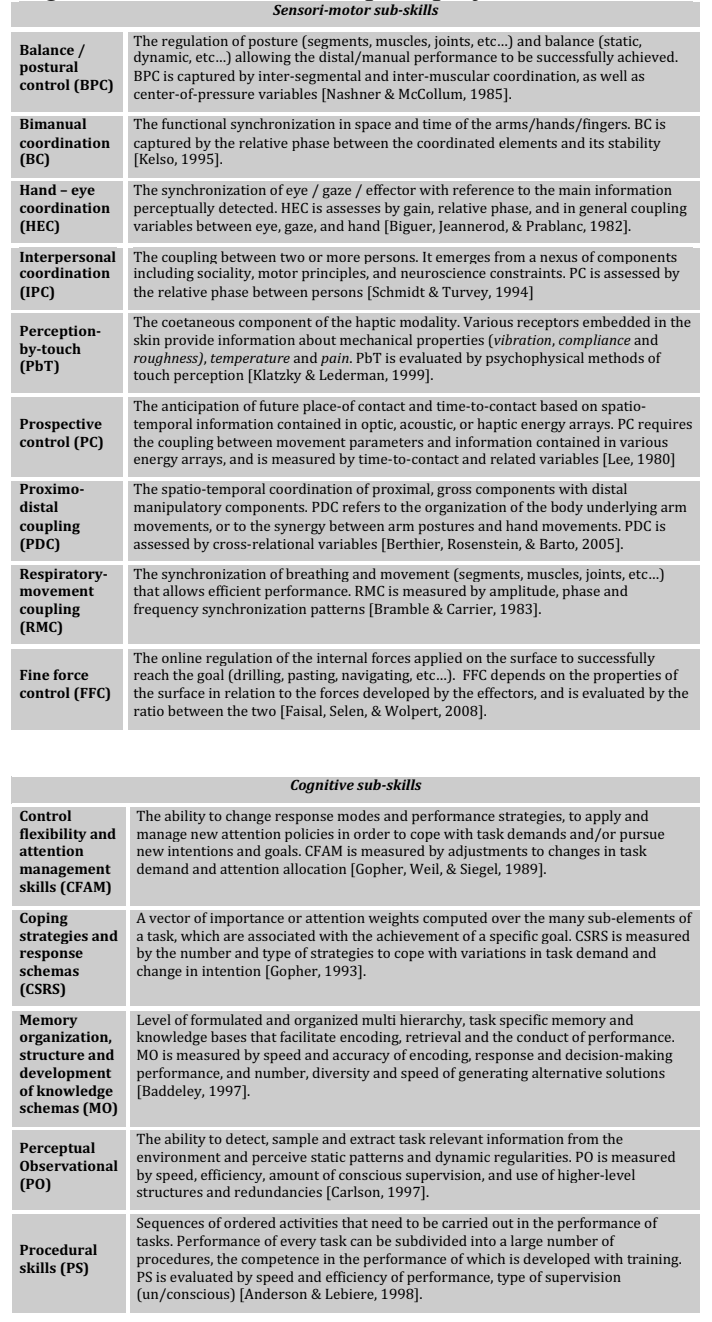

\section{Enactive learning and skill acquisition}

As mentioned in the previous section, the distinction between sensori-motor and cognitive sub-skills is pragmatically operational but partly arbitrary as the two categories are largely interdependent. This is because of the natural embodiment of cognitive phenomena into sensorimotor dynamics, and the possibility to enact highly cognitive phenomena using contemporary rendering techniques. Although there are several views on embodied cognition and enaction [6], the terms refers to the basic fact that cognition is largely for action, that off-line cognition (cognition decoupled from the environment) is largely dependent on the dynamics of body movements and behaviour, and that the acquisition of knowledge is largely realized by doing and interacting with the environment. Perceptual inputs 
for instance (e.g., vision) can elicit covert motor activity in the absence of any task demand. One illustration is judgments of whether a screwdriver is screwing or unscrewing, which have been shown to be faster when the orientation of the handle is consistent with the manual dominance of the observer [7]. Other illustrations come from brain imaging studies having shown that the perception of objects affords actions towards these objects with identical activity in cortical regions [8]. In the same vein, the fact that when individuals observe an action, a brain activation similar to the one arising when actually producing that action is generated in their premotor cortex [9], suggests that embodied cognition plays a role in representing and understanding the behaviour of conspecifics [10], such as in learning by imitation for instance. Hence perception is not solely a visual or auditory or tactile process, preceding symbolic representations of actions to be performed. What we perceive in the world is influenced not only by, for instance, optical and ocular-motor information, but also by our purposes, physiological state, and emotions. Perception and cognition are thus embodied; they relate body and goals to the opportunities and costs of acting in the environment [11]. In that respect, theories of embodied cognition and enaction largely rely on the influential work by Gibson in the last fifty years [12] calling for a mutual relation between information and movement, between perceiving and acting.

Virtual reality technology allows the enactment of new perception-action and cognitive components that are of importance for learning. The enactive interfaces develops within the SKILLS project represent a mean to enhance the conditions for carrying out intuitively manipulative procedures or for learning complex perceptuo-motor skills, to study the conditions for the user of "getting his hands inthere and acting" [13], to improve subjective (the feeling of 'being there') and objective (performatory) fidelity [14], and to evoke new affordances using virtual environments.

\section{Illustration: learning team rowing using VR}

The decomposition of a complex perceptuo-motor skill into functional sub-skills allows focusing virtual reality based training on specific elements. An example is given here in team rowing. When rowing in team, the difference in performance between two teams often depends on the ability of the athletes to row together in a highly synchronized way during the race [15]. For identical movement patterns, the highest speed of the boat is indeed obtained when rowers' movements are synchronized, i.e., when the continuous relative phase between rowers is null. By using the combination of virtual reality rendering techniques and real-time motion capturing, we [16] recently investigated whether it was possible to learn the specific interpersonal coordination sub-skill with a virtual teammate on an indoor rowing machine, and to transfer the acquired skill to synchronizing with a real teammate.

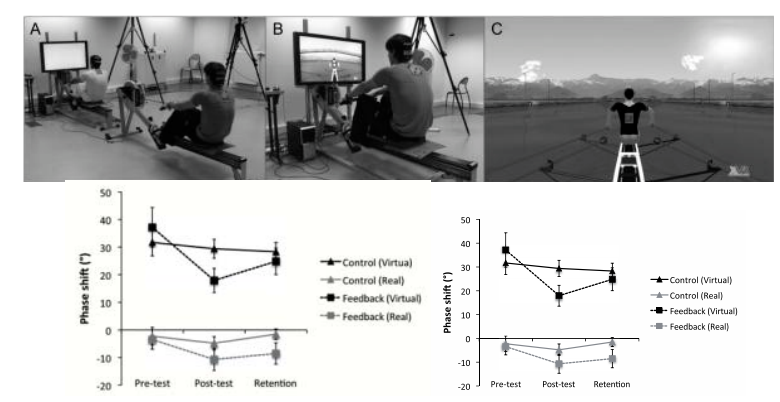

Figure 2. Up: A VR training protocol for learning team rowing showing real pre-test and post-test (A), training sessions with or without embodied on-line visual information about the between-rowers relative phase (B \& C); Bottom: Difference in coordination performance (left) and coordination variability (right) between a control group and feedback group at pre-test, post-test, and retention. Adapted from [16].

In our learning protocol, the synchronization was either spontaneous (no feedback other than the presence of the avatar), or increased by online visual information embodied on the avatar, giving real-time information about the between-bodies coordination (see Figure $2 \mathrm{~B} \& \mathrm{C}$ ). The results were straightforward and indicated that all participants improved their ability to synchronize with a real teammate (pre-post test comparison, see Figure 2A) following a training protocol with a virtual partner. However, learning was better for the participants that had the embodied feedback available

\section{References}

[1] B. G. Bardy, J. Lagarde, and Mottet. Dynamics of skill acquisition in multimodal technological environments. In Skills training in multimodal virtual environments, M. Bergamasco, B. G. Bardy, \& D. Gopher (Eds.), Boca Raton (FL, USA): Taylor \& Francis, in press.

[2] M. T. Turvey. Coordination. American 
Psychologist, 45, 938-953, 1980.

[3] F. Mussa-Ivaldi. Modular features of motor control and learning. Current Opinion in Neurobiology, 9, 713-717, 1999.

[4] S. Schaal, S. Kotosaka, and D. Sternad. Nonlinear dynamical systems as movement primitives. Humanoids2000, First IEEE-RAS International Conference on Humanoid Robots, CDProceedings, 2000.

[5] M. Bergamasco, B. G. Bardy and D. Gopher (Eds.). Skills training in multimodal virtual environments. Boca Raton (FL, USA): Taylor \& Francis, in press.

[6] F. J. Varela, E. Thompson and E. Rosch. The Embodied Mind: Cognitive Science and Human Experience. Cambridge, MA: The MIT Press, 1991.

[7] M. Wilson. Six views of embodied cognition. Psychonomic Bulletin and Review, 9, 625-636, 2002.

[8] C. De'Sperati and N. Stucchi. Recognising the motion of a graspable object is guided by handedness. Neuroreport, 8, 2761-2765, 1997.

[9] J. Grezes and J. Decety. Does visual perception of object afford action? Evidence from a neuroimaging study. Neuropsychologia, 40, 212-222, 2002.

[10] G. Buccino, F. Binkofski, G. R. Fink, L. Fadiga, L. Fogassi, V. Gallese, R. J. Seitz, K. Zilles, G. Rizzolatti and H. J. Freund. Action observation activates premotor and parietal areas in a somatotopic manner: an fMRI study. European Journal of Neuroscience, 13, 400-404, 2001.

[11] J. J. Gibson. The ecological approach to visual perception. Boston, Houghton Mifflin, 1979.

[12] J. Stewart, O. Gapenne, and E. Di Paolo (Eds). Enaction: Towards a New Paradigm for Cognitive Science. Cambridge, MIT Press, 2011.

[13] T. A. Stoffregen, B. G. Bardy, J. L. Smart, and R. J. Pagulayan. On the nature and evaluation of fidelity in virtual environments. In L.J. Hettinger \& M.W. Haas (Eds.), Virtual and adaptive environments: Applications, implications, and Human performance issues (pp. 111-128). Mahwah, NJ: Lawrence Erlbaum, 2003.

[14] H. Hill. Dynamics of coordination within elite rowing crews: evidence from force pattern analysis. Journal of Sports Sciences, 20, 101-117, 2002.

[15] M. Varlet, A. Filippeschi, G. Ben-sadoun, M. Ratto, L. Marin, E. Ruffaldi, and B. G. Bardy (2011). Learning team rowing coordination using virtual reality. Submitted. 